\title{
Operating injection lasers by fast square current pulses of variable amplitude
}

\author{
D. R. Armstrong, A. Katzir, and A. Yariv \\ California Institute of Technology, Pasadena, California 91125
}

(Received 12 March 1975)

\begin{abstract}
A simple solid state circuit was used to drive GaAs injection lasers by fast $(\sim 100$ nsec) square pulses of variable amplitude (0-25 A). The amplitudes of the current pulses and the corresponding emitted light pulses were measured by a dual peak detector circuit. Using these circuits we were able to plot automatically the current vs light curve and determine the threshold current of the laser diodes.
\end{abstract}

\section{INTRODUCTION}

Gallium arsenide injection lasers driven by fast current pulses may well serve as optical pulse generators for optical communication systems. In the course of studying GaAsGaAlAs distributed feedback injection lasers ${ }^{1}$ at low temperatures, we tried to transfer fast square current pulses along a transmission line to lasers mounted in a Dewar, and to measure accurately the amplitudes of the current pulse and the light pulse emitted by the laser.

In the testing of experimental injection lasers, a great deal of time can be saved if the plotting of light output versus current, or current versus voltage, can be done automatically on an $X-Y$ recorder, rather than by point-by-point procedures. Since these measurements must be made using short pulse techniques, the requirements for an automatic plotting system include a source of square current pulses of programmable amplitude and circuits that detect the peak values of the diode current and photodetector output and convert them to dc analogs for operating the recorder. The circuits described recently in the literature ${ }^{2-4}$ were inadequate for this application, and we report here some circuits that have given satisfactory results.

\section{PULSE GENERATOR}

The preferred characteristics of current pulses for testing injection lasers include the following:

(1) A short risetime to minimize preheating, preferably less than $10 \mathrm{nsec}$.

(2) A flat top, so that the amplitude is precisely known.

(3) A sufficiently narrow width to avoid excessive power dissipation, usually less than 200 nsec.

(4) Variable amplitude over a range of approximately $0.1-20 \mathrm{~A}$.

(5) Adequate repetition rate to meet the requirements of the rest of the system.

(6) Freedom from amplitude or time delay jitter.

Needless to say, perfection on all counts lies in the realm of wishful thinking, but an acceptable level of performance is possible with some compromises.

Of the various discrete devices used to pulse injection lasers, the avalanche transistor appears to have the best combination of characteristics. ${ }^{5}$ However, since a transistor will avalanche (if at all) only over a narrow range of collector voltage, thus providing a correspondingly narrow range of current into a fixed load, there is a problem of how to increase the current range.

Furthermore, the simplest method of obtaining a square pulse with an avalanche transistor is to discharge a charged transmission line through it, but in order to get the necessary current from a line, while staying within the voltage limitations of the transistor, the line impedance must be very low. For example, if the maximum collector voltage is $120 \mathrm{~V}$ (a representative value), a current of $20 \mathrm{~A}$ into a matched load requires an impedance of about $3 \Omega$. Higher currents may, of course, be obtained by operating the line into a load of less than its characteristic impedance, but the resulting voltage reversal at the end of the pulse is hazardous to GaAs diodes, which have a low reverse voltage tolerance. Ordinary silicon rectifiers are not fast enough to bypass these reversals satisfactorily; however, the recently available high speed Schottky barrier rectifiers are useful for this purpose.

Since there is little available information regarding the avalanche or pulse characteristics of commercial transistor types, the development of practical circuits of this kind must be done largely by empirical means.

Distributed-constant transmission lines with an impedance of a few ohms are difficult to prepare in the necessary lengths (approximately $9.5 \mathrm{~m} / 100 \mathrm{nsec}$ ), but at the sacrifice of some ideality of pulse shape, a lumped-constant network may be used. A form that works quite well consists simply of a ladder of ceramic disk capacitors soldered to a pair of parallel wires. The values and spacing of the capacitors can be adjusted experimentally to give a reasonably square pulse into a desired load resistance.

The general circuit of the pulse generator is shown in Fig. 1. The approach used is to charge a transmission line $\left(\mathrm{TL}_{2}\right)$ to the voltage necessary to give the desired current into the load, and to discharge it through a fast transistor $\left(\mathrm{Q}_{2}\right)$ that is rapidly driven into saturation by avalanching another transistor $\left(Q_{1}\right)$ into its base. Because of the low impedance level, the impedances of the transistors and connections are a not insignificant fraction of the load on the transmission line. Consequently, the pulse shape is influenced 


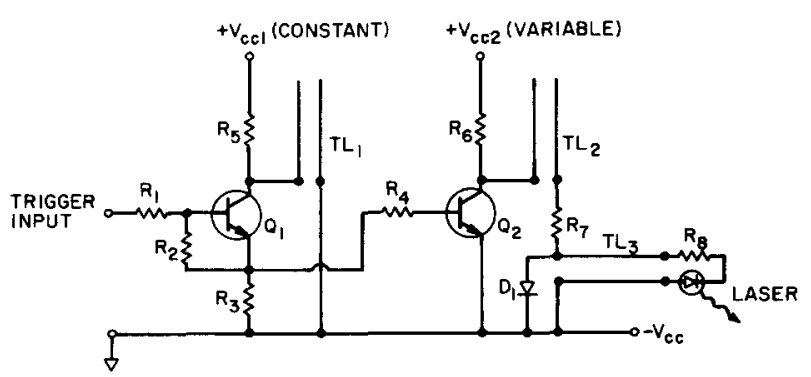

FIG. 1. Avalanche transistor pulse generator circuit. Component values are listed in the Appendix.

by a variety of factors, and some tailoring of the circuit is necessary.

It is found that the best pulse shape is obtained when the drive pulse from the avalanche transistor, $Q_{1}$, has the same width as the output pulse, so a transmission line $\left(\mathrm{TL}_{1}\right)$ is used as the charge storage element for that stage as well. It is also found that the output pulse is terminated most rapidly if the drive pulse is allowed to reverse, i.e., if $T_{1}$ is loaded by less than its characteristic impedance. This loading, and also the drive pulse amplitude, can be adjusted by means of $R_{3}$ and $R_{4}$.

$\mathrm{TL}_{1}$ must necessarily be charged to a voltage sufficient to avalanche $Q_{1}$, so the drive pulse to $Q_{2}$ has a constant amplitude, irrespective of the level of the output pulse. A result of this is that the shape of the output pulse is modified, at low current levels, by transients from the drive pulse. Consequently, the range of useful current levels obtainable from a given circuit is limited, the ratio of maximum to minimum currents typically being on the order of 15 or 20 to 1 . The needs of our program have been met by constructing two puse generators, one covering a range of 0.1-2 A for testing diodes cooled in liquid nitrogen, the other covering 1-20 A for room temperature tests. The low-current version uses two RG-58/U cables in parallel for each transmission line; the high-current one uses lumped-constant networks.

For operation of diodes at liquid nitrogen temperature, it is necessary to conduct the pulse from the generator down into a Dewar, which requires another transmission line of the correct impedance ( $\mathrm{TL}_{3}$ ) if the pulse is not to be severely distorted. A suitable material for such lines is thin polyimide sheet with copper cladding on both sides, such as is manufactured for flexible printed circuits. The laminate may be

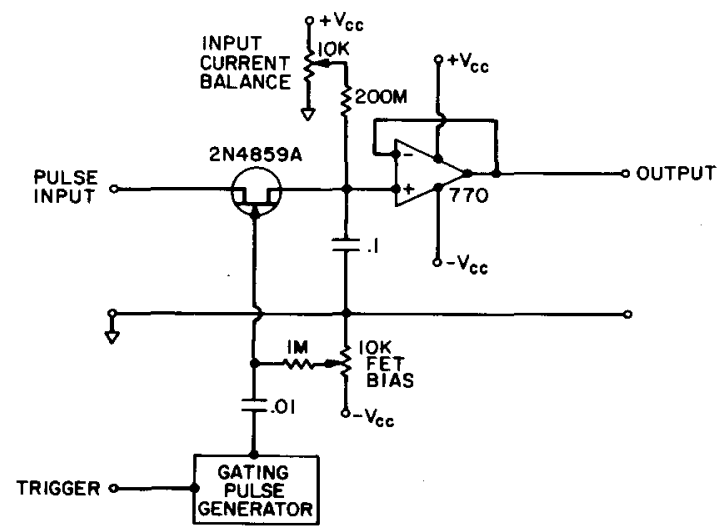

Fig. 2. Peak detector circuit. cut into strips, whose dimensions determine the characteristic impedance. The following formula may be used as an approximate guide:

$$
Z=337(1 / k)^{\frac{1}{2}} b / a
$$

where $Z$ is the characteristic impedance, $a$ the conductor width, $b$ the dielectric thickness, and $k$ the dielectric constant.

Because of the nonlinear nature of a diode, it may be desirable to add some series resistance at the end of the strip to improve the matching, otherwise the strip may tend to ring. At high currents, where additional series resistance cannot be afforded, ringing may be partially damped out by parallel resistance at the generator end of the strip.

Several transistor types have been tested for suitability in this circuit, and the best we have found to date is the $2 \mathrm{~N} 3725$, which allows a short risetime, high current, and reliable avalanche behavior. For lower currents, the 2N2222A works well. Some increase in maximum current and decrease in risetime result from connecting two transistors in parallel in the $\mathrm{Q}_{2}$ position.

Since the output pulse amplitude is proportional to the supply voltage for $\mathrm{Q}_{2}$, the amplitude may be swept, for use in an automatic plotting system, by using a slow voltage ramp.

The repetition rate is dependent only on the transistor dissipation, and a duty factor of $10^{-4}(1 \mathrm{kHz}$ at $100 \mathrm{nsec}$ width) presents no difficulty. A pulse generator with an output of about $4 \mathrm{~V}$ is sufficient to trigger the circuit, and the triggering delay has negligible jitter.

The amplitude of the current pulse is conveniently measured by means of a miniature wide-band current probe, mounted on one of the leads of the injection laser, and connected to an oscilloscope.

\section{PEAK DETECTOR}

A sample-and-hold technique is required for recording the amplitude of the pulses, since the voltage levels and duty factor are too small to allow the use of diode peak detectors. A simple approach that gives satisfactory results is to charge a capacitor from the pulse through a field-effect transistor, which is gated on for a period totally contained within the pulse width.

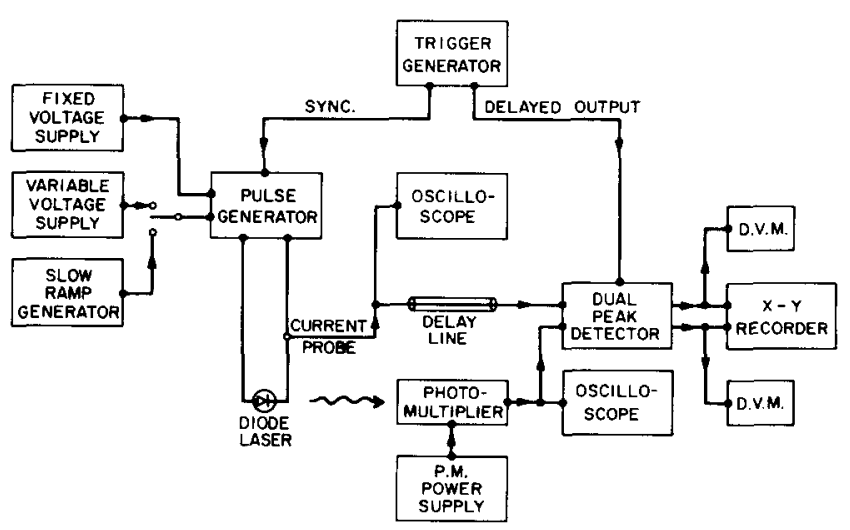

FIG. 3. Block diagram of the automatic plotting system. 
Fig. 4. Current pulse measured through an RCA SG2001 laser diode. Vertical scale: $5 \mathrm{~A} /$ div. Horizontal scale: $20 \mathrm{nsec} /$ div.

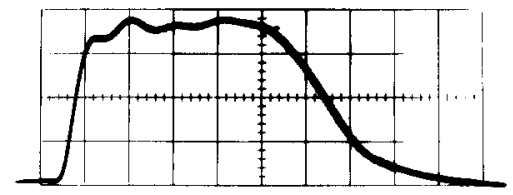

The ratio of off-state to on-state resistance of a choppertype FET such as the $2 \mathrm{~N} 4859 \mathrm{~A}$ is so high that at a duty factor of $10^{-4}$, the decay of capacitor voltage between pulses due to FE'T leakage alone is negligible. However, care must be taken to minimize other sources of leakage if reasonable accuracy is to be expected. A high quality capacitor and high input resistance voltage follower are, of course, important.

Figure 2 shows the essentials of a circuit that has permitted measurement of pulse amplitude with an error of less than $10 \%$, at 100 nsec pulse width and 500 pulses/sec. The gating pulse for the FET is conveniently supplied by a TTL monostable multivibrator, followed by an open-collector buffer to amplify the multivibrator output to a level of about $10 \mathrm{~V}$. Its width is adjusted to be somewhat less than that of the pulse to be measured. There are several options in the choice of a voltage follower, and presumably an operational amplifier with FET inputs would be an improvement over the type 770 .

The pulse amplitude that can be measured with this circuit is limited to about $\pm 2 \mathrm{~V}$, since above this value, the voltage on the storage capacitor begins to in terfere significantly with the FET bias, and the accuracy decreases. Where higher levels are to be measured, an attenuator may be used at the input.

For use with an automatic plotting system, where two peak detectors are required, it is convenient to assemble both circuits on the same board, and operate both FETs with the same biasing and gate-pulsing circuits.

\section{PLOTTING SYSTEM}

A block diagram of a system for plotting light output versus laser current is shown in Fig. 3. Since, in this case, both peak detectors are operated with the same gating circuit, it is necessary to delay the current probe pulse to compensate for the delay of the light signal from the injec-

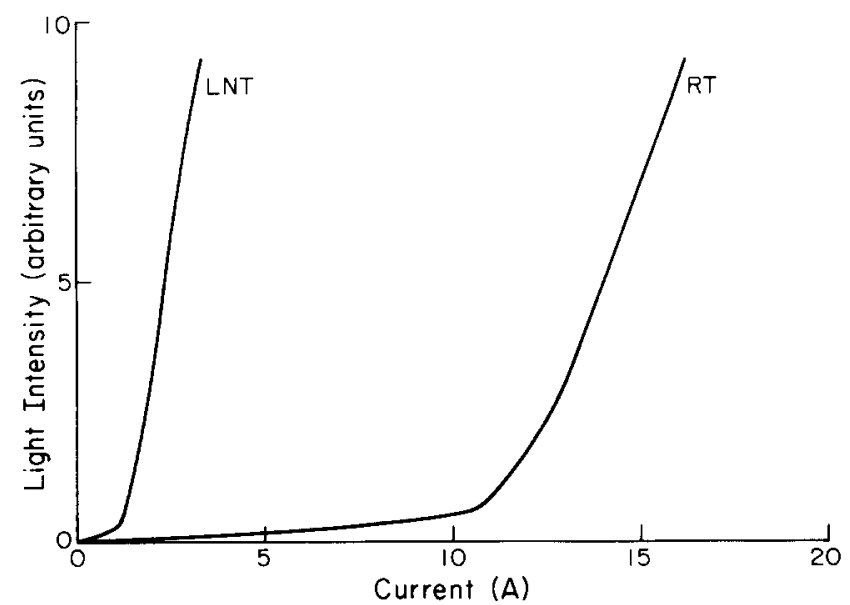

FIG. 5. Automatic plotting of light vs current for RCA SG2001 laser diode at 300 and $77 \mathrm{~K}$.

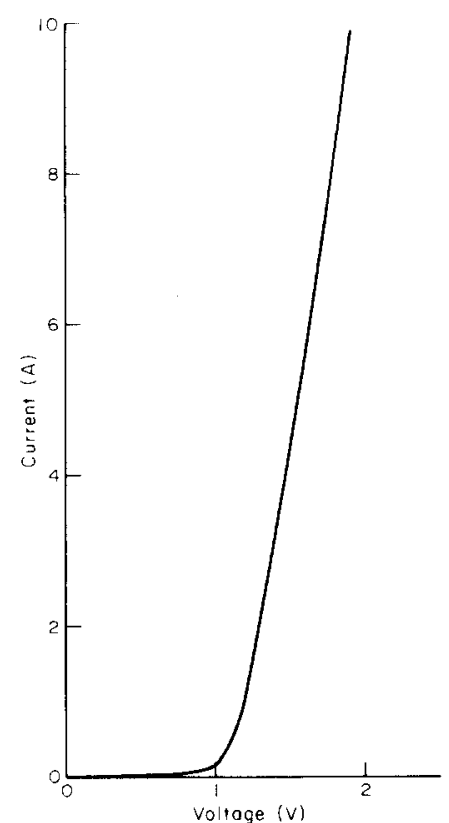

tion laser, so that they both arrive at the peak detectors simultaneously. Alternatively, the peak detectors may be designed for separate triggering; then two trigger generators with individual delays would be required.

Adjustment of the delays is easily done by noting, on the oscilloscopes, when the gating transients fall within the current and light pulses.

A useful source of a slow ramp for the laser pulse generator is the sawtooth output from the time base of a Tektronix series 500 oscilloscope.

\section{RESULTS}

We checked our system by measuring the properties of a commercial RCA SG2001 laser diode. A typical current pulse through this diode is shown in Fig. 4. The light vs current curves were measured at room temperature $(300 \mathrm{~K})$ and at liquid nitrogen temperature $(77 \mathrm{~K})$, and the results are shown in Fig. 5. The threshold current at $300 \mathrm{~K}$ was found to be $11 \mathrm{~A}$, and at $77 \mathrm{~K}$ about $1 \mathrm{~A}$. We also measured the current vs voltage curve at $300 \mathrm{~K}$ in a similar manner, as shown in Fig. 6. From this curve we deduced that the forward resistance of the diode is $r_{f}=\Delta V / \Delta I \sim 0.1 \Omega$, in the range $1-10 \mathrm{~A}$.

\section{APPENDIX}

Values of circuit components for constructed pulsers are as follows:

(A) Current range $0.1-2 \mathrm{~A}$

$\begin{array}{lccc}\mathrm{R}_{1}, \mathrm{R}_{2}, \mathrm{R}_{4} & 100 \Omega & \mathrm{R}_{6} & 22 \mathrm{k} \Omega \\ \mathrm{R}_{3} & 22 \Omega & \mathrm{R}_{7} & 15 \Omega \\ \mathrm{R}_{5} & 47 \mathrm{k} \Omega & \mathrm{R}_{8} & 4.3 \Omega\end{array}$

$\mathrm{TL}_{1}, \mathrm{TL}_{2}$ each made from 2 lengths of RG-58/U cable, $9.45 \mathrm{~m}$ long, connected in parallel (for 100 nsec pulses).

$\mathrm{TL}_{3} \quad 30.5 \times 1.27 \mathrm{~cm}$ strip of laminated sheet, $0.127 \mathrm{~mm}$ polyimide clad with $0.025 \mathrm{~mm}$ copper. 
$D_{1} \quad$ Not used.

$\mathrm{Q}_{1}, \mathrm{Q}_{2} \quad 2 \mathrm{~N} 3725$

(B) Current range $1-20 \mathrm{~A}$

$\begin{array}{ll}\mathrm{R}_{1}, \mathrm{R}_{2} & 100 \Omega \\ \mathrm{R}_{3} & \text { open circuit } \\ \mathrm{R}_{4} & 1.4 \Omega \\ \mathrm{R}_{5} & 47 \mathrm{k} \Omega \\ \mathrm{R}_{6} & 22 \mathrm{k} \Omega \\ \mathrm{R}_{7} & \text { short circuit } \\ \mathrm{R}_{8} & \text { short circuit } \\ \mathrm{TL}_{1} & 8 \text { ea } 0.001 \mu \mathrm{F} \text { disk capacitors spaced } 2.5 \mathrm{~cm} \\ & \text { apart on wires spaced } 1 \mathrm{~cm} \text { apart. } \\ \mathrm{TL}_{2} & 7 \text { ea } 0.002 \mu \mathrm{F} \text { disk capacitors spaced } 1.25 \mathrm{~cm} \\ & \text { apart on wires spaced } 1 \mathrm{~cm} \text { apart. }\end{array}$

$\mathrm{TL}_{3} \quad$ As in (A).

$\mathrm{D}_{1} \quad$ TRW type DSR5050

Q1 $2 \mathrm{~N} 3725$

$\mathrm{Q}_{2} \quad 2$ ea $2 \mathrm{~N} 3725$ in parallel.

Tower supplies :
$\mathrm{V}_{\text {ec1 }}$
$130 \mathrm{~V} \mathrm{dc}$
$\mathrm{V}_{\mathrm{co} 2}$
$0-130 \mathrm{~V} \mathrm{dc}$

${ }^{1}$ M. Nakamura, K. Aiki, J. Umeda, A. Katzir, A. Yariv, and H. W. Yen, IEEE J. Quantum. Electron. QE-11, 436 (1975).

${ }^{2}$ J. Vanderwall, W. V. Hattery, and Z. G. Sztenkay, IEEE J. Quantum Electron. QE-10, 570 (1974).

${ }^{3}$ J. R. Andrews, Rev. Sci. Instrum. 45, 22 (1974).

${ }^{4} \mathrm{~W}$. Herden and S. Metz, IEEE J. S Jid-State Circuits SC-8, 247 (1973).

${ }^{5}$. L. Carroll, Electronics 44 (26), 59 (1971). 